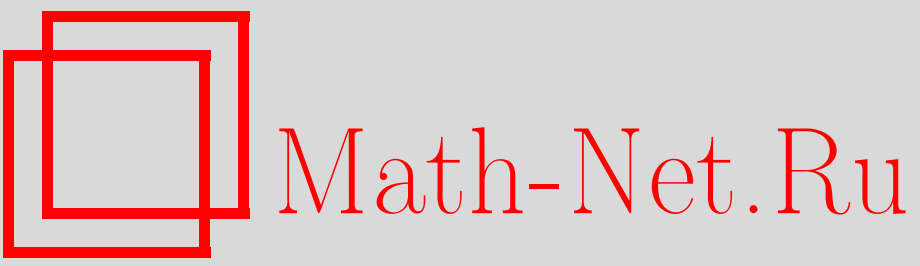

Я. Г. Синай, О распределении максимума дробного броуновского движения, УМH, 1997, том 52, выпуск 2, 119-138

DOI: https://doi.org/10.4213/rm822

Использование Общероссийского математического портала Math-Net.Ru подразумевает, что вы прочитали и согласны с пользовательским соглашением

http://www.mathnet.ru/rus/agreement

Параметры загрузки:

IP: 54.198 .64 .247

26 апреля 2023 г., $14: 16: 17$ 


\title{
О РАСПРЕДЕЛЕНИИ МАКСИМУМА ДРОБНОГО БРОУНОВСКОГО ДВИЖЕНИЯ
}

\author{
Я. Г. СинАЙ \\ СОДЕРЖАНИЕ
}

$\S 1$. Определение дробного броуновского движения. Основной резуль-

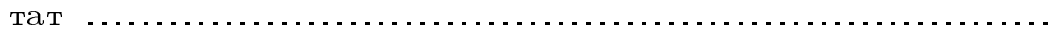

$\S 2$. Явные формулы для $m\left(C_{\rho}\right)$ и $\sigma(s, T)$. Асимптотическое поведение

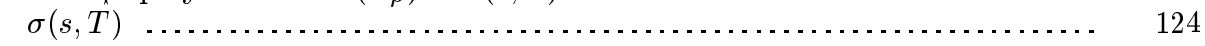

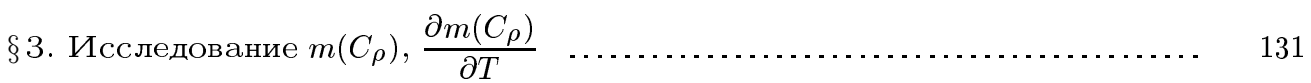

$\S 4$. Окончание доказательства основной теоремы. Заключительные за-

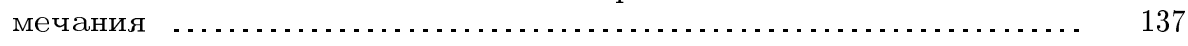

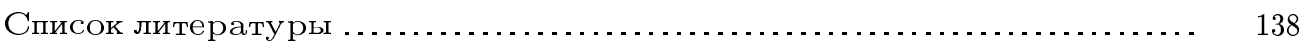

\section{$\S$ 1. Определение дробного броуновского движения. Основной результат}

Дробное броуновское движение (ДБД) представляет собой гауссовский процесс со стационарными приращениями, распределение вероятностной которого инвариантно относительно групшы масштабных преобразований. Именно по этой причине ДБД возникает во многих приложениях. Впервые ДБД появилось в работе А. Н. Колмогорова [1] и, несколько позднее, в работах П. Леви [2] и Б. Мандельброта [3]. По поводу определения и основных свойств ДБД см. [3], [4]. Фрактальная структура множества нулей ДБД была исследована в [4], более поздние результаты см. в [5], [6].

В этой работе мы изучаем распределение максимума ДБД. Начнем с построения ДБД.

Простейший способ, связывающий ДБД с методом ренормгрупшы в статистической механике и с теорией автомодельных распределений (см. [7]-[9]), состоит в следующем. Рассмотрим гауссовскую комплексную ортогональную меру $\Phi(\Lambda), \Lambda \in \mathscr{B}\left(\mathbb{R}^{1}\right)$, для которой $\Phi(-\Lambda)=\overline{\Phi(\Lambda)}, \mathscr{B}\left(\mathbb{R}^{1}\right)$ обозначает борелевскую $\sigma$-алгебру на $\mathbb{R}^{1}$, и

$$
\mathrm{E}|\Phi(\Lambda)|^{2}=C \int_{\Lambda} \frac{d \lambda}{|\lambda|^{\alpha+2}}
$$

в случае $\operatorname{dist}(\Lambda, 0)>0$. Здесь $\alpha$ - основной параметр ДБД, меняюшийся в пределах $-1<\alpha<1$. Обычное броуновское движение отвечает $\alpha=0$. Значение константы $C$ не играет роли и поэтому в дальнейшем $C=1$. 
Введем “приращения"

$$
\xi\left(t^{\prime}, t^{\prime \prime}\right)=\int_{-\infty}^{\infty}\left(e^{i \lambda t^{\prime}}-e^{i \lambda t^{\prime \prime}}\right) d \Phi(\lambda), \quad t^{\prime}>t^{\prime \prime}
$$

При рассматриваемых $\alpha$ последний интеграл имеет смысл как обычный стохастический интеграл и дает вешественнозначную гауссовскую случайную величину со средним 0 и дисперсией

$$
\mathrm{D} \xi\left(t^{\prime}, t^{\prime \prime}\right)=\int_{-\infty}^{\infty} \frac{\left|e^{i \lambda t^{\prime}}-e^{i \lambda t^{\prime \prime}}\right|^{2}}{|\lambda|^{\alpha+2}} d \lambda=\int_{-\infty}^{\infty} \frac{d \lambda\left|1-e^{i \lambda\left(t^{\prime}-t^{\prime \prime}\right)}\right|^{2}}{|\lambda|^{\alpha+2}}=C_{0}\left(t^{\prime}-t^{\prime \prime}\right)^{\alpha+1} .
$$

По этой причине распределение $\xi\left(t^{\prime}+t, t^{\prime \prime}-t\right)$ не зависит от $t$ и $\xi\left(t^{\prime}, t^{\prime \prime}\right)+\xi\left(t^{\prime \prime}, t^{\prime \prime \prime}\right)=$ $\xi\left(t^{\prime}, t^{\prime \prime \prime}\right)$ с вероятностью 1.

Далее мы изучаем случайньй процесс $\xi(t)=\xi(t, 0), t>0$. Только при $\alpha=0$ этот процесс является марковским. В общем случае отвечаюшее ему распределение вероятностей инвариантно относительно группы масштабных преобразований $S(r)$, где $(S(r) \xi)(t)=\frac{1}{r \frac{\alpha+1}{2}} \xi(r t)$. Иными словами, распределение вероятностей семейства случайных величин $\left\{\xi_{r}(t)\right\}$ не зависит от $r$. Заеле и Пачке (см. [10]) доказали, что группа $\{S(r)\}$ эргодична.

Нетрудно установить, что с вероятностью 1 реализации $\xi(t)$ удовлетворяют условию Гёльдера с показателем $\frac{\alpha+1}{2}-\varepsilon$ и не удовлетворяют никакому условию Гёльдера с показателем $\frac{\alpha+1}{2}+\varepsilon$. Здесь $\varepsilon>0-$ произвольно. Ниже в этой работе мы рассматриваем только $0<\alpha<1$. Реализации таких процессов более гладкие, чем реализации обычного броуновского движения. По этой причине здесь не возникает хорошо известных трудностей, связанных с формулой Ито в теории стохастических дифференщиальных уравнений, и, в частности, для соответствующих стохастических интегралов справедлива обычная формула интегрирования по частям.

По построению $\xi(0)=0$. Введем случайную величину $\nu_{T}=\max _{0 \leqslant t \leqslant T} \xi(t)$. Для броуновского движения $(\alpha=0)$ хорошо известно (см., например, $[1])$, что при любом $a>0$

$$
\mathrm{P}\left\{\nu_{T} \leqslant a\right\} \sim \frac{\text { const }}{T^{1 / 2}}, \quad T \rightarrow \infty .
$$

Здесь и в дальнейшем обозначение const используется для постоянных, значение которых несушественно, но может зависеть от $\alpha$.

Цель этой работы - обобщить соотношение (1) на обшие ДБД с $0<\alpha<1$. Введем случайную величину $\tau_{a}$, равную моменту первого пересечения уровня $a$. Тогда $P(T)=\mathrm{P}\left\{\nu_{T}<a\right\}=\mathrm{P}\left\{\tau_{a}>T\right\}$. Мы выведем для функции $P(T)$ интегральное уравнение. Будем действовать так же, как в случае обычного броуновского движения. Рассмотрим измеримое разбиение $\rho$ пространства элементарных событий $\Omega$, элемент которого $C_{\rho}$ есть пара $(s ; \xi(t), 0 \leqslant t \leqslant s), \xi(t)$ есть реализация нашего процесса до момента $s$, причем $\xi(t)<a$ при $t<s$ и $\xi(s)=a$. Иными словами, элемент $C_{\rho}$ разбиения $\rho$ задается реализацией $\xi(t)$ до момента $s$ первого достижения ею уровня $a$. Мы можем теперь написать

$$
\mathrm{P}\{\xi(T)>a\}=\int_{A_{T}} d P\left\{C_{\rho}\right\} \mathrm{P}\left\{\xi(T)>a \mid C_{\rho}\right\}
$$


где интегрирование идет по множеству $A_{T}$ тех элементов $C_{\rho}$, где $s \leqslant T$. Формула (2) есть формула полной вероятности, справедливая без каких-либо дополнительных предположений. Далее мы примем без доказательства, что ДБД обладает "строго марковским свойством", согласно которому условное распределение $\xi(T)-\xi(s)$ при условии $C_{\rho}$ такое же, как и для неслучайного $s$, т.е. имеет вид гауссовского распределения, дисперсия которого не зависит от условия и есть функция только $T$ и $s$, а вся зависимость от условия заключена в условном математическом ожидании $m\left(C_{\rho}\right)$, которое представляет собой линейньй функционал $\xi(t), 0 \leqslant t \leqslant s$, такой же, как и при неслучайном $s$. Строго марковское свойство можно доказать обычным методом путем дискретизации времени и предельным переходом при стремлении шага дискретизации к нулю. Мы можем написать теперь

$$
\begin{aligned}
\mathrm{P}\left\{\xi(T)>a \mid C_{\rho}\right\} & =\mathrm{P}\left\{\xi(T)-\xi(s)-m\left(C_{\rho}\right)>-m\left(C_{\rho}\right)\right\} \\
& =\frac{1}{\sqrt{2 \pi \sigma(s, T)}} \int_{-m\left(C_{\rho}\right)}^{\infty} e^{-\frac{u^{2}}{2 \sigma(s, T)}} d u=\frac{1}{\sqrt{2 \pi}} \int_{-\frac{m\left(C_{\rho}\right)}{\sqrt{\sigma(s, T)}}}^{\infty} e^{-\frac{u^{2}}{2}} d u
\end{aligned}
$$

Возврашаясь к (2), получаем

$$
\mathrm{P}\{\xi(T)>a\}=\int_{0}^{T} d P_{\tau_{a}}(s)\left(\frac{1}{2}-\Phi(s, T)\right),
$$

где $P_{\tau_{a}}$ есть функщия распределения случайной величины $\tau_{a}$, т.е. "интеграл по множеству элементов разбиения $\rho$ с данными $s "$,

$$
\Phi(s, T)=\int d P\left(C_{\rho}, s\right) \int_{0}^{-\frac{m\left(C_{\rho}\right)}{\sqrt{\sigma(s, T)}}} \frac{1}{\sqrt{2 \pi}} e^{-\frac{u^{2}}{2}} d u .
$$

Внешнее интегрирование происходит по условному распределению $C_{\rho}$ при заданном $s$.

В $\S 2$ мы выведем явные выражения для $m\left(C_{\rho}\right)$ и $\sigma(s, T)$, из которых будет следовать диффенцируемость $\Phi(s, T)$ по $T$. Используя равенство $\mathrm{P}\{\xi(T)>a\}=$ $\frac{1}{2}-\mathrm{P}\{0<\xi(T)<a\}$ и дифференцируя обе части (3) по $T$, получим, что функция распределения $P_{\tau_{a}}$ имеет плотность, которую мы обозначим $p_{\tau_{a}}(x)$, и эта плотность удовлетворяет интегральному уравнению типа Вольтерра:

$$
r(T)=p_{\tau_{a}}(T)\left(\frac{1}{2}-\Phi(T, T)\right)+2 \int_{0}^{T} p_{\tau_{a}}(s) d s \Phi_{T}^{\prime}(s, T),
$$

где $r(T)=-\frac{d}{d T} \mathrm{P}\{0<\xi(T)<a\}$ есть известная функция $T$, про которую легко доказать, что $r(T) \sim \frac{\text { const }}{T^{\frac{\alpha+3}{2}}}$ при $T \rightarrow \infty$. Величина $\Phi(T, T)$ понимается как предел $\Phi(T, T)=\lim _{s \uparrow T} \Phi(s, T)$. Мы увидим, что он отличен от нуля. Это утверждение следует также из того, что при малых $T-s$ наилучший прогноз $m\left(C_{\rho}\right)$ имеет порядок $\sqrt{\sigma(s, T)}$, что само по себе неудивительно. Мь исследуем в дальнейшем поведение $\Phi(T, T)$ при $T \rightarrow \infty$. 
Удобно выделить малые $s$ отдельно и переписать (5) следующим образом:

(6) $\left(\frac{1}{2}-\Phi(T, T)\right)^{-1} r_{1}(T)=p_{\tau_{a}}(T)+2 \int_{1}^{T} p_{\tau_{a}}(s) d s \Phi_{T}^{\prime}(s, T)\left(\frac{1}{2}-\Phi(T, T)\right)^{-1}$,

где $r_{1}(T)=r(T)-2 \int_{0}^{1} p_{\tau_{a}}(s) d s \Phi_{T}^{\prime}(s, T)$. В дальнейшем считаем $T \geqslant 2$.

Ясно, что асимптотика $p_{\tau_{a}}(T)$ определяется поведением

$$
\Phi_{T}^{\prime}(s, T)=\mathrm{E}_{s, a} \frac{1}{\sqrt{2 \pi}} \exp \left\{-\frac{m^{2}\left(C_{\rho}\right)}{2 \sigma(s, T)}\right\} \cdot \frac{\partial}{\partial T} \frac{m\left(C_{\rho}\right)}{\sqrt{\sigma(s, T)}} .
$$

Здесь и далее $\mathrm{E}_{s, a}$ обозначает условное математическое ожидание при условии $\tau=s$, т.е. интеграл по множеству элементов $C_{\rho}$ с данными $s$ и $a$. При дальнейшем анализе интегрального уравнения (6) нам понадобится асимптотика условных математических ожиданий $\mathrm{E}_{s, a} \xi(u), \mathrm{E}_{s, a}(\xi(s)-\xi(u))$. Поскольку при любом $s>0$ процесс $\xi_{s}^{(1)}(t)=\xi(t s) / s^{\frac{\alpha+1}{2}}$ имеет такое же распределение вероятностей, что и $\xi(t)$, то

$$
\mathrm{E}_{s, a}|\xi(u)|=s^{\frac{1+\alpha}{2}} \cdot \mathrm{E}_{1, a / s} \frac{1+\alpha}{2}\left|\xi\left(\frac{u}{s}\right)\right|=s^{\frac{1+\alpha}{2}} h_{1}\left(\frac{u}{s}, s\right) .
$$

В этом условном математическом ожидании интегрирование происходит по множеству реализаций $\xi(t)$, для которых $\xi(0)=0, \xi(1)=a / s^{\frac{1+\alpha}{2}}$ и $\xi(t)<a / s^{\frac{1+\alpha}{2}}$ при $0 \leqslant s<1$. При $s \rightarrow \infty$ это интегрирование переходит в интегрирование по множеству траекторий, для которых $\xi(0)=\xi(1)=0, \xi(t)<0$ при $0<t<1$.

Сформулируем теперь предположение о функщии $h_{1}(v, s)$, которое представляется весьма правдоподобньм, но доказательством которого мы в данньй момент не располагаем.

Н1) При $v<\frac{1}{2}$

$$
h_{1}(v, s)=v^{\frac{1+\alpha}{2}} g_{1}(v, s),
$$

где $\left|g_{1}(v, s)\right| \leqslant$ const, причем const не зависит от $\alpha$.

$\mathrm{C}$ помошью $\mathrm{H} 1)$ можем написать $\mathrm{E}_{s, a}|\xi(u)|=u^{\frac{1+\alpha}{2}} g_{1}\left(\frac{u}{s}, s\right)$.

Аналогичным образом, при любом $s$ процесс $\xi_{s}^{(2)}(t)=\frac{\xi(s)-\xi(s t)}{s^{\frac{1+\alpha}{2}}}$ при $0 \leqslant t \leqslant 1$ имеет распределение вероятностей, не зависящее от $s$. Поэтому

$$
\mathrm{E}_{s, a}(\xi(s)-\xi(u))=s^{\frac{1+\alpha}{2}} h_{2}\left(\frac{s-u}{s}, s\right)
$$

и $h_{2}$ имеет предел при $s \rightarrow \infty, \frac{u}{s}$ фиксировано.

Н2) При $v \leqslant \frac{1}{2}$

$$
h_{2}(v, s)=v^{\frac{1+\alpha}{2}} g_{2}(v, s)
$$

и $g_{2}(v, s)$ равномерно ограничена, $g_{2}(v, s) \leqslant$ const, причем const не зависит от $\alpha$.

$\mathrm{C}$ помошь $\mathrm{H} 2)$ можем написать

$$
\mathrm{E}_{s, a}(\xi(s)-\xi(u))=(s-u)^{\frac{1+\alpha}{2}} g_{2}\left(\frac{s-u}{s}, s\right)
$$


Н3) Существует $\lim _{u \rightarrow 0} g_{2}(u, s)=g_{2}(0, s)$ и

$$
\left|g_{2}(v, s)-g_{2}(0, s)\right| \leqslant \operatorname{const}\left(\frac{v}{s}\right)^{\gamma}
$$

где $\gamma>0$ и const не зависят от $\alpha$.

При этих предположениях мы покажем в $\S 2$ и $\S 3$, что $\Phi_{t}(s, T)$ допускает оценки:

$\left.\mathrm{B}_{1}\right)$ при $0<s \leqslant T / 2$

$$
\left|\Phi_{T}(s, T)\right| \leqslant \frac{\text { const } \cdot \alpha \cdot s^{\frac{1-\alpha}{2}}}{T^{\frac{3-\alpha}{2}}}
$$

$\left.\mathrm{B}_{2}\right)$ при $T / 2 \leqslant s \leqslant T$

$$
\left|\Phi_{T}(s, T)\right| \leqslant \frac{\text { const } \cdot \alpha}{(T-s)^{1-\gamma} s^{\gamma}} .
$$

Здесь и далее const не зависит от $\alpha$.

Из В $\left._{1}\right)$, В $_{2}$ ) вытекает, что

$$
\int_{0}^{T}\left|\Phi_{T}(s, T)\right| d s \leqslant \text { const } \cdot \alpha .
$$

Кроме того, $\left|r_{1}(T)\right| \leqslant$ const $\cdot \alpha \cdot T^{-\frac{3-\alpha}{2}}$. При достаточно малых $\alpha$ мы можем написать решение (6) в виде ряда

$$
\begin{aligned}
p(T)= & r_{1}(T)\left(\frac{1}{2}-\Phi(T, T)\right)^{-1}-2 \int_{1}^{T} r_{1}\left(s_{0}\right) \Phi_{T}^{\prime}\left(s_{0}, T\right)\left(\frac{1}{2}-\Phi(T, T)\right)^{-2} d s_{0} \\
& +2^{2} \int_{1}^{T} d s_{0} \Phi_{T}^{\prime}\left(s_{0}, T\right) \int_{1}^{s_{0}} d s_{1} r_{1}\left(s_{1}\right) \Phi_{s_{0}}^{\prime}\left(s_{1}, s_{0}\right)\left(\frac{1}{2}-\Phi(T, T)\right)^{-3} \\
& -2^{3} \int_{1}^{T} \Phi_{T}^{\prime}\left(s_{0}, T\right) d s_{0} \int_{1}^{s_{0}} \Phi_{s_{0}}^{\prime}\left(s_{1}, s_{0}\right) d s_{1} \\
& \times \int_{1}^{s_{1}} \Phi_{s_{1}}^{\prime}\left(s_{2}, s_{1}\right) r_{1}\left(s_{2}\right) d s_{2}\left(\frac{1}{2}-\Phi(T, T)\right)^{-4}+\cdots
\end{aligned}
$$

Написанный ряд сходится в $L^{1}(0, T)$ при любом $T \geqslant 0$ и для достаточно малых $\alpha$. Нетрудно также показать, что решение уравнения (6) единственно в классе функций с $\int_{0}^{T}|p(s)| d s \leqslant 1$ и решение допускает при больших $T$ оценку

$$
p_{\tau_{a}}(T) \leqslant \frac{\text { const } \cdot \alpha}{T^{\frac{3-\alpha}{2}+b(\alpha)}}
$$

где $|b(\alpha)| \leqslant$ const $\cdot \alpha$. Мы можем теперь сформулировать основной результат этой статьи.

ОСНОВНАЯ ТЕОРЕМА. При достаточно мальх $\alpha$ плотность $p_{\tau_{a}}(T)$ удовлетворяет неравенству (8).

В $\S 2$ мы исследуем поведение $\sigma(s, T)$, а в $\S 3$ - поведение $m\left(C_{\rho}\right), m(s)=\mathrm{E}_{s} m(C \rho)$. Заметим, что $\S 3$ составляет наиболее трудную часть работы. Здесь мы используем 
ряд сокрашений, которые вытекают из наших предположений Н1)-Н3). Окончание доказательства, ряд замечаний и гипотез изложены в $\$ 4$.

Я благодарю А. А. Рузмайкину за помош в проведении ряда выгислений и Г. М. Заславского за полезные обсуждения. Я благодарен также Г. М. Молчану за многочисленные критические замечания, способствовавшие улучшению текста. Благодарю также NSF (грант DMS 9404437) и фонд РФФИ (грант № 96-01-00377) за финансовую поддержку.

\section{$\S$ 2. Явные формулы для $m\left(C_{\rho}\right)$ и $\sigma(s, T)$. \\ Асимптотическое поведение $\sigma(s, T)$}

Запишем прирашение $\xi(T)-\xi(s)$ в виде

$$
\xi(T)-\xi(s)=\int_{0}^{s} K(u) d \xi(u)+\xi^{\prime}(T, s),
$$

где $\xi^{\prime}(T, s)$ не зависит от всех $\xi\left(t^{\prime}, t^{\prime \prime}\right), 0 \leqslant t^{\prime \prime}<t^{\prime} \leqslant s$. Стохастический интеграл в последнем выражении есть $m\left(C_{\rho}\right)$. Он существует при определенных условиях на функцию $K(u)$, которые мы сейчас не обсуждаем и которые заведомо выполнены в нашем случае.

Лемма 1. $\mathrm{E}(d \xi(u) d \xi(v))=\frac{C_{0} \alpha(\alpha+1)}{|u-v|^{1-\alpha}} d u d v$ при некоторой постоянной $C_{0}=$ $C_{0}(\alpha)$, для которой $\lim _{\alpha \rightarrow 0} C_{0}(\alpha)>0$.

Утверждение леммы справедливо при всех $\alpha,-1<\alpha<1$. Однако только при $\alpha>0$ написанное ядро интегрируемо по каждой переменной. Этим, в частности, объясняется условие $\alpha>0$, которое было введено в самом начале этой статьи.

Доказательство основано на обшей формуле для $\mathrm{E}(\xi(u) \xi(v))$, которую мы сейчас вьведем. Имеем при $u>v>0$

$$
\begin{aligned}
\mathrm{E}(\xi(u) \xi(v))= & \int_{-\infty}^{\infty} \frac{\left(e^{i \lambda u}-1\right)\left(e^{-i \lambda v}-1\right)}{|\lambda|^{\alpha+2}} d \lambda \\
= & \lim _{\varepsilon \rightarrow 0} \int_{|\lambda|>\varepsilon} \frac{\left(e^{i \lambda u}-1\right)\left(e^{-i \lambda v}-1\right)}{|\lambda|^{\alpha+2}} d \lambda \\
= & \lim _{\varepsilon \rightarrow 0} \int_{\varepsilon}^{\infty} \frac{\left[e^{i \lambda(u-v)}+e^{-i \lambda(u-v)}\right]-\left[e^{i \lambda u}+e^{-i \lambda u}\right]-\left[e^{i \lambda v}+e^{-i \lambda v}\right]+2}{\lambda^{\alpha+2}} d \lambda \\
= & \lim _{\varepsilon \rightarrow 0}\left[(u-v)^{\alpha+1} \int_{\varepsilon(u-v)}^{\infty} \frac{e^{i \mu}+e^{-i \mu}}{\mu^{\alpha+2}} d \mu-u \int_{\varepsilon u}^{\infty} \frac{e^{i \mu}+e^{-i \mu}}{\mu^{\alpha+2}} d \mu\right. \\
& \left.-v \int_{\varepsilon v}^{\infty} \frac{e^{i \mu}+e^{-i \mu}}{\mu^{\alpha+2}} d \mu+\frac{2}{(\alpha+1) \varepsilon^{\alpha+1}}\right] \\
= & \lim _{\varepsilon \rightarrow 0}\left[( u - v ) ^ { \alpha + 1 } \left(\int_{\varepsilon(u-v)}^{1} \frac{e^{i \mu}+e^{-i \mu}-2}{\mu^{\alpha+2}} d \mu\right.\right. \\
& \left.+\frac{2}{\alpha+1}\left(\frac{1}{\varepsilon^{\alpha+1}(u-v)^{\alpha+1}}-1\right)+\int_{1}^{\infty} \frac{e^{i \mu}+e^{-i \mu}}{\mu^{\alpha+2}} d \mu\right) \\
& -u^{\alpha+1}\left(\int_{\varepsilon u}^{1} \frac{e^{i \mu}+e^{-i \mu}-2}{\mu^{\alpha+2}} d \mu+\frac{2}{\alpha+1}\left(\frac{1}{\varepsilon^{\alpha+1} u^{\alpha+1}}-1\right)\right. \\
& \left.\left.+\int_{1}^{\infty} \frac{e^{i \mu}+e^{-i \mu}}{\mu^{\alpha+2}} d \mu\right)+\frac{2}{(\alpha+1) \varepsilon^{\alpha+1}}\right]
\end{aligned}
$$




$$
\begin{aligned}
= & {\left[u^{\alpha+1}+v^{\alpha+1}-(u-v)^{\alpha+1}\right] } \\
& \times\left[\frac{2}{\alpha+1}-\int_{0}^{1} \frac{e^{i \mu}+e^{-i \mu}-2}{\mu^{\alpha+2}} d \mu-\int_{0}^{\infty} \frac{e^{i \mu}+e^{-i \mu}-2}{\mu^{\alpha+2}} d \mu\right] \\
= & C_{0}\left[u^{\alpha+1}+v^{\alpha+1}-(u-v)^{\alpha+1}\right] .
\end{aligned}
$$

Из этой формулы следует, что $\mathrm{E}(\xi(u)-\xi(v))^{2}=2 C_{0}(u-v)^{\alpha+1}$.

ДокАЗАТЕЛЬСТво ЛЕммы 1. Имеем

$$
\mathrm{E}(\xi(u)-\xi(v))=\frac{\partial^{2} \mathrm{E}(\xi(u) \xi(v))}{\partial u \partial v} d u d v=C_{0}(\alpha+1) \alpha(u-v)^{\alpha-1} .
$$

Лемма доказана.

ЛЕмма 2.

$$
\mathrm{E}(\xi(T)-\xi(s)) d \xi(u)=C_{2}\left((T-u)^{2}-(s-u)^{\alpha}\right) d u
$$

при $C_{2}=C_{0}(\alpha+1)$.

ДокАЗАТЕльСтво. Имеем

$$
\begin{aligned}
\mathrm{E}(\xi(T)-\xi(s)) d \xi(u) & =d u \frac{\partial}{\partial u} \mathrm{E}(\xi(T)-\xi(s)) \xi(u) \\
& =C_{0}(\alpha+1)\left((T-u)^{\alpha}-(s-u)^{\alpha}\right)=C_{2}\left((T-u)^{\alpha}-(s-u)^{\alpha}\right) .
\end{aligned}
$$

Лемма доказана.

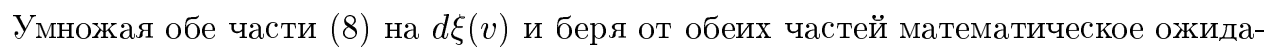
ние, получаем, что $K(u)$ выражается через решение интегрального уравнения Абеля (см. [2])

$$
\begin{gathered}
\left((T-v)^{\alpha}-(s-v)^{\alpha}\right)=\alpha \int_{0}^{s}(K(T, u)-K(s, u)) \frac{1}{|u-v|^{1-\alpha}} d u, \\
K(u)=K(T, u)-K(s, u) .
\end{gathered}
$$

Уравнению (10) посвящена огромная литература, и его решение может быть выражено в явном виде. Мы воспользуемся формулой (30.84) из книги Самно, Килбаса и Маричева [11], согласно которой

$$
\begin{aligned}
K(x, u)= & \frac{\operatorname{tg} \frac{\alpha \pi}{2}}{2 \pi \alpha} \frac{d}{d u} \int_{0}^{s} \frac{\operatorname{sign}(u-t)}{|u-t|^{\alpha}}(x-t)^{\alpha} d t \\
& +\frac{\sin ^{2} \frac{\alpha \pi}{2}}{2 \pi^{2} \alpha} \frac{d}{d u} \int_{0}^{s} M(u, t)[t(s-t)]^{-\frac{\alpha}{2}}(x-t)^{\alpha} d t \\
= & K_{1}(u, x)+K_{2}(u, x),
\end{aligned}
$$

где

$$
M(u, t)=\int_{0}^{s} \frac{\sqrt{t(s-t)}-\sqrt{y(s-y)}}{t-y} \cdot \frac{d y}{|u-y|^{\alpha}[y(s-y)]^{\frac{1-\alpha}{2}}} .
$$


Через $K_{1}, K_{2}$ обозначены отдельные слагаемые в (11). Таким образом,

$$
\begin{aligned}
m\left(C_{\rho}\right) & =\frac{\operatorname{tg} \frac{\alpha \pi}{2}}{2 \pi \alpha} m_{1}\left(C_{\rho}\right)+\frac{\sin ^{2} \frac{\alpha \pi}{2}}{2 \pi^{2} \alpha} m_{2}\left(C_{\rho}\right), \\
m_{1}\left(C_{\rho}\right) & =\int_{0}^{s}\left(K_{1}(u, T)-K_{1}(u, s)\right) d \xi(u), \\
m_{2}\left(C_{\rho}\right) & =\int_{0}^{s}\left(K_{2}(u, T)-K_{2}(u, s)\right) d \xi(u), \\
\frac{\partial m\left(C_{\rho}\right)}{\partial T} & =\frac{\operatorname{tg} \frac{\alpha \pi}{2}}{2 \pi \alpha} \cdot \frac{\partial m_{1}\left(C_{\rho}\right)}{\partial T}+\frac{\sin ^{2} \frac{\alpha \pi}{2}}{2 \pi^{2} \alpha} \cdot \frac{\partial m_{2}\left(C_{\rho}\right)}{\partial T}, \\
\frac{\partial m_{i}\left(C_{\rho}\right)}{\partial T} & =\int_{0}^{s} \frac{\partial K_{i}(u, T)}{\partial T} d \xi(u), \quad i=1,2 .
\end{aligned}
$$

Мы вьведем сейчас выражение для $\sigma(s, T)$. Поскольку $\xi^{\prime}(T, s)$ не зависит от всех $\xi\left(t^{\prime}, t^{\prime \prime}\right), 0 \leqslant t^{\prime \prime}<t^{\prime} \leqslant s$, то

$$
\mathrm{E}(\xi(T)-\xi(s))^{2}=\mathrm{E}\left[\int_{0}^{s}\left(K_{1}(u, T)-K_{1}(u, s)\right) d \xi(u)\right]^{2}+\mathrm{E}\left(\xi^{\prime}(T, s)\right)^{2} .
$$

Поскольку $\mathrm{E}(\xi(T)-\xi(s))^{2}=2 C_{0}(T-s)^{\alpha+1}$, то

$$
\sigma(s, T)=\mathrm{E}\left(\xi^{\prime}(T, s)\right)^{2}=2 C_{0}(T-s)^{\alpha+1}-\bar{\sigma}(s, T),
$$

где

$$
\begin{aligned}
\bar{\sigma}(s, T) & =\mathrm{E}\left[\int_{0}^{T}(K(u, T)-K(u, s)) d \xi(u)\right]^{2} \\
& =\int_{0}^{s} \int_{0}^{s}\left(K\left(u_{1}, T\right)-K\left(u_{1}, s\right)\right)\left(K\left(u_{2}, T\right)-K\left(u_{2}, s\right)\right) \frac{1}{\left|u_{1}-u_{2}\right|^{1-\alpha}} d u_{1} d u_{2} \\
& =C_{0}(1+\alpha) \int_{0}^{s}\left((T-u)^{\alpha}-(s-u)^{\alpha}\right)(K(T, u)-K(s, u)) d u
\end{aligned}
$$

так как (см. (9))

$$
\int_{0}^{s}(K(u, T)-K(u, s)) \frac{1}{|u-v|^{1-\alpha}} d u=\frac{1}{\alpha}\left((T-v)^{\alpha}-(s-v)^{\alpha}\right) .
$$

Таким образом, для $\bar{\sigma}(s, T)$ мы имеем представление

$$
\bar{\sigma}(s, T)=\bar{\sigma}_{1}(s, T)+\bar{\sigma}_{2}(s, T),
$$

где

$$
\bar{\sigma}_{1}(s, T)=C_{0}(\alpha+1) \frac{\operatorname{tg} \frac{\alpha \pi}{2}}{2 \pi \alpha} I_{1}, \quad \bar{\sigma}_{2}(s, T)=C_{0}(\alpha+1) \frac{\sin ^{2} \frac{\alpha \pi}{2}}{2 \pi^{2} \alpha^{2}} I_{2},
$$

и

$$
\begin{gathered}
I_{1}=\int_{0}^{s}\left((T-u)^{\alpha}-(s-u)^{\alpha}\right) d u \frac{d}{d u} \int_{0}^{s} \frac{\operatorname{sign}(u-t)\left((T-t)^{\alpha}-(s-t)^{\alpha}\right)}{|u-t|^{\alpha}} d t \\
I_{2}=\int_{0}^{s}\left((T-u)^{\alpha}-(s-u)^{\alpha}\right) d u \frac{d}{d u} \int_{0}^{s} M(u, t)[t(s-t)]^{-\frac{\alpha}{2}}\left[(T-t)^{\alpha}-(s-t)^{\alpha}\right] d t \\
M(u, t)=\int_{0}^{s} \frac{\sqrt{t(s-t)}-\sqrt{y(s-y)}}{t-y} \cdot \frac{d y}{|u-y|^{\alpha}[y(s-y)]^{\frac{1-\alpha}{2}}} .
\end{gathered}
$$


Как уже отмечалось, мы можем считать $T \geqslant 2$, поскольку при мальх $T$ все оценки тривиальны.

Рассмотрим вначале $I_{1}$.

После интегрирования по частям получим:

$$
\begin{aligned}
I_{1}= & (T-s)^{\alpha} \int_{0}^{s} \frac{(T-t)^{\alpha}-(s-t)^{\alpha}}{(s-t)^{\alpha}} d t+\left(T^{\alpha}-s^{\alpha}\right) \int_{0}^{s} \frac{(T-t)^{\alpha}-(s-t)^{\alpha}}{t^{\alpha}} d t \\
& +\alpha \int_{0}^{s}\left((T-u)^{\alpha-1}-(s-u)^{\alpha-1}\right) d u \int_{0}^{u} \frac{(T-t)^{\alpha}-(s-t)^{\alpha}}{(u-t)^{\alpha}} d t \\
& -\alpha \int_{0}^{s}\left((T-u)^{\alpha-1}-(s-u)^{\alpha-1}\right) d u \int_{u}^{s} \frac{(T-t)^{\alpha}-(s-t)^{\alpha}}{(t-u)^{\alpha}} d t .
\end{aligned}
$$

Производя еше несколько раз интегрирование по частям во внутренних и внешних интегралах, можем написать

$$
\begin{aligned}
I_{1}= & 2\left(T^{\alpha}-s^{\alpha}\right) \int_{0}^{s} \frac{(T-u)^{\alpha}-(s-u)^{\alpha}}{u^{\alpha}} d u \\
& -2 \alpha \int_{0}^{s}\left((T-u)^{\alpha-1}-(s-u)^{\alpha-1}\right) d u \int_{u}^{s} \frac{(T-t)^{\alpha}-(s-t)^{\alpha}}{(t-u)^{\alpha}} d t .
\end{aligned}
$$

Положим $x=T / s$. Ясно, что $x \geqslant 1$. Имеем

$$
\begin{aligned}
I_{1}= & s^{1+\alpha}\left[2\left(x^{\alpha}-1\right) \int_{0}^{1} \frac{(x-u)^{\alpha}-(1-u)^{\alpha}}{u^{\alpha}} d u\right. \\
& \left.-2 \alpha \int_{0}^{1}\left((x-u)^{\alpha-1}-(1-u)^{\alpha-1}\right) d u \int_{u}^{1} \frac{(x-t)^{\alpha}-(1-t)^{\alpha}}{(t-u)^{\alpha}} d t\right] .
\end{aligned}
$$

Далее, с помошью замен во внутренних интегралах $\frac{t-u}{1-u}=t_{1}$ и $\frac{t-u}{x-u}=t_{1}$, соответственно, получим

$$
\begin{aligned}
\int_{0}^{1}\left((x-u)^{\alpha-1}-(1-u)^{\alpha-1}\right) d u \int_{u}^{1} \frac{(x-t)^{\alpha}-(1-t)^{\alpha}}{(t-u)^{\alpha}} d t \\
=\int_{0}^{1}\left((x-u)^{\alpha-1}-(1-u)^{\alpha-1}\right) d u \\
\quad \times\left[(u-1) \int_{0}^{1} \frac{(1-t)^{\alpha}}{t^{\alpha}} d t-(x-u) \int_{0}^{\frac{1-u}{x-u}} \frac{(1-t)^{\alpha}}{t^{\alpha}} d t\right] \\
=\int_{0}^{1}\left((x-u)^{\alpha-1}-(1-u)^{\alpha-1}\right) d u \\
\quad \times\left[(x-1) \int_{0}^{1} \frac{(1-t)^{\alpha}}{t^{\alpha}} d t-(x-u) \int_{\frac{1-u}{x-u}}^{1} \frac{(1-t)^{\alpha}}{t^{\alpha}} d t\right] .
\end{aligned}
$$

Наконец, замена $1-u=(x-1) w$ дает для последнего интеграла выражение

$$
\begin{aligned}
\int_{0}^{1}\left((x-u)^{\alpha-1}-\right. & \left.(1-u)^{\alpha-1}\right) d u \int_{u}^{1} \frac{(x-t)^{\alpha}-(1-t)^{\alpha}}{(t-u)^{\alpha}} d t \\
=(x-1)^{\alpha+1}\{ & \int_{0}^{\frac{1}{x-1}}\left[w^{\alpha-1}-(1+w)^{\alpha-1}\right] \\
& \left.\times\left[(1+w) \int_{\frac{w}{1+w}}^{1} \frac{(1-t)^{\alpha}}{t^{\alpha}} d t-\int_{0}^{1} \frac{(1-t)^{\alpha}}{t^{\alpha}} d t\right] d w\right\} .
\end{aligned}
$$


Заметим, что выражение в фигурных скобках имеет конечньй предел при $x \rightarrow 1$. Далее,

$$
\begin{aligned}
\int_{0}^{1} \frac{(x-u)^{\alpha}-(1-u)^{\alpha}}{u^{\alpha}} d u & =x \int_{0}^{\frac{1}{x}} \frac{(1-u)^{\alpha}}{u^{\alpha}} d u-\int_{0}^{1} \frac{(1-u)^{\alpha}}{u^{\alpha}} d u \\
& =(x-1) \int_{0}^{1} \frac{(1-u)^{\alpha}}{u^{\alpha}} d u-\int_{\frac{1}{x}}^{1} \frac{(1-u)^{\alpha}}{u^{\alpha}} d u .
\end{aligned}
$$

Таким образом,

$$
\begin{aligned}
& I_{1}=s^{1+\alpha}\left\{2\left(x^{\alpha}-1\right)\right. {\left[(x-1) \int_{0}^{1} \frac{(1-u)^{\alpha}}{u^{\alpha}} d u-\int_{\frac{1}{x}}^{1} \frac{(1-u)^{\alpha}}{u^{\alpha}} d u\right] } \\
&+2 \alpha(x-1)^{\alpha+1} \int_{0}^{\frac{1}{x-1}}\left(\frac{1}{w^{1-\alpha}}-\frac{1}{(1+w)^{1-\alpha}}\right) \\
&\left.\times\left(\int_{0}^{1} \frac{(1-t)^{\alpha}}{t^{\alpha}} d t-(1-w) \int_{\frac{w}{1+w}}^{1} \frac{(1-t)^{\alpha}}{t^{\alpha}} d t\right) d w\right\} .
\end{aligned}
$$

Сформулируем итог всех этих вычислений в виде следующей леммы.

Лемма 3. Имеет место равенство

$$
I_{1}=(T-s)^{\alpha+1} \varphi_{1}\left(\frac{T}{s}\right),
$$

причем $\varphi_{1}(x)$ имеет конечный предел при $x \rightarrow 1$. При малых $\alpha$ и любом $x$ функиия $\varphi_{1}\left(\frac{T}{s}\right)=O(\alpha)$.

То, что $I_{1}$ должно иметь подобное представление, можно было предвидеть и из общих соображений.

Исследуем теперь $\frac{\partial I_{1}}{\partial T}$. Из (16) имеем при $x=T / s$

$$
\begin{aligned}
\frac{\partial I_{1}}{\partial T}= & 2 \alpha T^{\alpha-1} s \int_{0}^{1} \frac{(x-u)^{\alpha}-(1-u)^{\alpha}}{u^{\alpha}} d u \\
& +2 \alpha\left(T^{\alpha}-s^{\alpha}\right) \int_{0}^{1} \frac{(x-u)^{\alpha-1}}{u^{\alpha}} d u \\
& +2(1-\alpha) s^{\alpha} \int_{0}^{1}(x-u)^{\alpha-2} d u \int_{u}^{1} \frac{(x-t)^{\alpha}-(1-t)^{\alpha}}{t^{\alpha}} d t \\
& -2 \alpha s^{\alpha} \int_{0}^{1}\left((x-u)^{\alpha-1}-(1-u)^{\alpha-1}\right) d u \int_{u}^{1} \frac{(x-t)^{\alpha-1}}{(t-u)^{\alpha}} d t \\
= & 2 \alpha T^{\alpha-1} s J_{1}+\left(T^{\alpha}-s^{\alpha}\right) J_{2}+2(1-\alpha) s^{\alpha} J_{3}+2 \alpha s^{\alpha} J_{4} .
\end{aligned}
$$

С помошю простых замен переменных получаем

$$
\begin{aligned}
& J_{1}=(x-1) \int_{0}^{1} \frac{(1-u)^{\alpha}}{u^{\alpha}} d u-x \int_{\frac{1}{x}}^{1} \frac{(1-u)^{\alpha}}{u^{\alpha}} d u, \\
& J_{2}=\int_{0}^{1} \frac{(x-u)^{\alpha-1}}{u^{\alpha}} d u=\int_{0}^{\frac{1}{x}} \frac{(1-u)^{\alpha-1}}{u^{\alpha}} d u .
\end{aligned}
$$


Рассмотрим интеграл $J_{3}$, где

$$
\begin{aligned}
J_{3} & =\int_{0}^{1}(x-u)^{\alpha-2} d u \int_{u}^{1} \frac{(x-t)^{\alpha}-(1-t)^{\alpha}}{u^{\alpha}} d t \\
& =\int_{0}^{1}(x-u)^{\alpha-2} d u\left(\int_{u}^{1} \frac{(x-t)^{\alpha}}{(t-u)^{\alpha}} d t-\int_{u}^{1} \frac{(1-t)^{\alpha}}{(t-u)^{\alpha}} d t\right) .
\end{aligned}
$$

После замены $t_{1}=\frac{t-u}{1-u}$ во внутреннем интеграле получим

$$
J_{3}=\int_{0}^{1}(x-u)^{\alpha-2} d u(1-u)\left[\int_{0}^{1} \frac{\left(1-t_{1}\right)^{\alpha}}{t_{1}^{\alpha}} d t_{1}-\frac{x-u}{1-u} \int_{0}^{\frac{1-u}{x-u}} \frac{\left(1-t_{1}\right)^{\alpha}}{t_{1}^{\alpha}} d t_{1}\right] .
$$

Во внешнем интеграле произведем замену $\frac{x-u}{1-u}$. Тогда

$$
\begin{aligned}
J_{3} & =-(x-1)^{\alpha} \int_{x}^{\infty} \frac{u_{1}^{\alpha-2} d u}{\left(u_{1}-1\right)^{\alpha+1}}\left[\int_{0}^{1} \frac{\left(1-t_{1}\right)^{\alpha}}{t_{1}^{\alpha}} d t_{1}-u_{1} \int_{0}^{\frac{1}{u_{1}}} \frac{\left(1-t_{1}\right)^{\alpha}}{t_{1}^{\alpha}} d t\right] \\
& =-(x-1)^{\alpha} \int_{x}^{\infty} \frac{u_{1}^{\alpha-2} d u}{\left(u_{1}-1\right)^{\alpha}}\left[\int_{0}^{1} \frac{\left(1-t_{1}\right)^{\alpha}}{t_{1}^{\alpha}} d t_{1}-u_{1} \int_{\frac{1}{u_{1}}}^{1} \frac{\left(1-t_{1}\right)^{\alpha}}{t_{1}^{\alpha}} d t\right] .
\end{aligned}
$$

Аналогичным образом для последнего интеграла $J_{4}$ в $(16)$ замена во внутреннем интеграле $\frac{t-u}{1-u}=t_{1}$ дает

$$
\begin{aligned}
J_{4} & =\int_{0}^{1}\left((1-u)^{\alpha-1}-(x-u)^{\alpha-1}\right) d u \int_{u}^{1} \frac{(x-t)^{\alpha-1}}{(t-u)^{\alpha}} d t \\
& =\int_{0}^{1}\left((1-u)^{\alpha-1}-(x-u)^{\alpha-1}\right) d u \int_{0}^{\frac{1-u}{x-u}} \frac{\left(1-t_{1}\right)^{\alpha}}{t_{1}^{\alpha}} d t_{1} .
\end{aligned}
$$

Теперь во внешнем интеграле сделаем замену $\frac{x-u}{x-1}=u_{1}$. В результате получим

$$
J_{4}=(x-1)^{\alpha} \int_{1}^{\frac{x}{x-1}}\left(\left(u_{1}-1\right)^{\alpha-1}-u_{1}^{\alpha-1}\right) d u_{1} \int_{0}^{\frac{u_{1}-1}{u_{1}}} \frac{\left(1-t_{1}\right)^{\alpha-1}}{t_{1}^{\alpha}} d t_{1} .
$$

Мыможем теперь сформулировать результат наших вычислений в виде следующей леммы.

Лемма 4. Имеет место представление

$$
\frac{\partial I_{1}}{\partial T}=(T-s)^{\alpha+1} \varphi_{2}\left(\frac{T}{s}\right),
$$

әде $\varphi_{2}(x)$ - ограниченная функиия при $x>1$, имеющая конечный предел при $x \rightarrow 1$.

Рассмотрим теперь

$$
I_{2}=\int_{0}^{s}\left((T-u)^{\alpha}-(s-u)^{\alpha}\right)\left(K_{2}(T, u)-K_{2}(s, u)\right) d u
$$


где

$$
K_{2}(z, u)=\frac{d}{d u} \int_{0}^{s} M(u, t)[t(s-t)]^{-\frac{\alpha}{2}}(z-t)^{\alpha} d t
$$

и

$$
M(u, t)=\int_{0}^{s} \frac{\sqrt{t(s-t)}-\sqrt{y(s-y)}}{t-y} \cdot \frac{d y}{|u-y|^{\alpha}[y(s-y)]^{\frac{1-\alpha}{2}}} ;
$$

интеграл $I_{2}$ только численным множителем отличается от

$$
\bar{\sigma}_{2}(s, T)=\frac{C_{0}(\alpha+1) \sin ^{2} \frac{\alpha \pi}{2}}{2 \pi^{2} \alpha^{2}} \int_{0}^{s}\left((T-u)^{\alpha}-(s-u)^{\alpha}\right)\left(K_{2}(T, u)-K_{2}(s, u)\right) d u .
$$

Сделаем вначале масштабное преобразование $u=u_{1} s, y=y_{1} s, t=t_{1} s$. Тогда мы получим при $x=T / s$

$$
\begin{aligned}
I_{2}= & s^{\alpha+1} \int_{0}^{1}\left(\left(x-u_{1}\right)^{\alpha}-\left(1-u_{1}\right)^{\alpha}\right) \frac{d}{d u_{1}} \int_{0}^{1} d t\left(\left(x-t_{1}\right)^{\alpha}-\left(1-t_{1}\right)^{\alpha}\right) \\
& \times \int_{0}^{1} \frac{1-t_{1}-y_{1}}{\sqrt{t_{1}\left(1-t_{1}\right)}+\sqrt{y_{1}\left(1-y_{1}\right)}} \cdot \frac{d y_{1}}{\left|u_{1}-y_{1}\right|^{\alpha}\left[y_{1}\left(1-y_{1}\right)\right]^{\frac{1-\alpha}{2}}} .
\end{aligned}
$$

Последний двойной интеграл стремится к конечным пределам при $u_{1} \rightarrow 0, u_{1} \rightarrow 1$. Действительно, при $u_{1} \rightarrow 0$ сделаем замену $y_{1}=u_{1} y_{2}$. Имеем

$$
\begin{gathered}
\int_{0}^{\frac{1}{u_{1}}} \frac{1-t_{1}-u_{1} y_{2}}{\sqrt{t_{1}\left(1-t_{1}\right)}+\sqrt{u} \sqrt{y_{2}\left(1-u_{1} y_{2}\right)}} \cdot \frac{u_{1}^{\frac{1-\alpha}{2}} d y_{2}}{\left|1-y_{2}\right|^{\alpha} y_{2}^{\frac{1-\alpha}{2}}\left(1-u_{1} y_{2}\right)^{\frac{1-\alpha}{2}}} \\
\sim \sqrt{\frac{1-t_{1}}{t_{1}}} \cdot u^{\frac{1-\alpha}{2}} \int_{0}^{\frac{1}{u_{1}}} \frac{d y_{2}}{y_{2}^{\frac{1-\alpha}{2}}\left|1-y_{2}\right|^{\alpha}} \sim \operatorname{const} \sqrt{\frac{1-t_{1}}{t_{1}}} .
\end{gathered}
$$

При $u \rightarrow 1$ этот интеграл ведет себя как const $\sqrt{\frac{t_{1}}{1-t_{1}}}$. Таким образом,

$$
\begin{aligned}
I_{2}= & \alpha s^{\alpha+1} \int_{0}^{1}\left(\left(x-u_{1}\right)^{\alpha-1}-\left(1-u_{1}\right)^{\alpha-1}\right) d u_{1} \int_{0}^{1} d t_{1}\left(\left(x-t_{1}\right)^{\alpha}-\left(1-t_{1}\right)^{\alpha}\right) \\
& \times \int_{0}^{1} \frac{1-t_{1}-y_{1}}{\sqrt{t_{1}\left(1-t_{1}\right)}+\sqrt{y_{1}\left(1-y_{1}\right)}} \cdot \frac{d y_{1}}{\left|u_{1}-y_{1}\right|^{\alpha}\left[y_{1}\left(1-y_{1}\right)\right]^{\frac{1-\alpha}{2}}} .
\end{aligned}
$$

Нетрудно показать, что при $x \rightarrow \infty$ интеграл $I_{2} \sim$ const $T^{s+1}\left(\frac{s}{T}\right)^{2-\alpha}$. Наоборот, при $x \rightarrow 1$

$$
I_{2}=(T-s)^{\alpha+1} \varphi_{3}\left(\frac{T}{s}\right),
$$

причем $\varphi_{3}(x)$ стремится к положительному пределу при $x \rightarrow 1$. Точно так же рассматривается производная $\frac{\partial I_{2}}{\partial T}$, для которой получается представление

$$
\frac{\partial I_{2}}{\partial T}=(T-s)^{\alpha} \varphi_{4}\left(\frac{T}{s}\right)
$$

где $\varphi_{4}(x)$ стремится к положительному пределу при $x \rightarrow 1$. Сформулируем окончательный итог всех вычислений этого параграфа. 
ТеОРема 1. Имеет место представление

$$
\sigma(s, T)=(T-s)^{\alpha+1} \psi_{1}\left(\frac{T}{s}\right), \quad \frac{\partial \sigma(s, T)}{\partial T}=(T-s)^{\alpha} \psi_{2}\left(\frac{T}{s}\right)
$$

где $\psi_{1}(x), \psi_{2}(x)$ - ограниченнье функиии, имеющие положительньй предел при $x \rightarrow 1$.

§. Исследование $m\left(C_{\rho}\right), \frac{\partial m\left(C_{\rho}\right)}{\partial T}$

Структура и свойства $m\left(C_{\rho}\right)$ более сложные. Мы воспользуемся формулой $(12)$. Рассмотрим вначале

$$
K_{1}(u, T)-K_{1}(u, s)=\frac{d}{d u} \int_{0}^{s} \frac{\operatorname{sign}(u-t)}{|u-t|^{\alpha}}\left((T-t)^{\alpha}-(s-t)^{\alpha}\right) d t
$$

Интегрирование по частям дает

$$
\begin{aligned}
\int_{0}^{s} & \frac{\operatorname{sign}(u-t)\left((T-t)^{\alpha}-(s-t)^{\alpha}\right) d t}{|u-t|^{\alpha}} \\
= & \int_{0}^{u} \frac{(T-t)^{\alpha}-(s-t)^{\alpha}}{(u-t)^{\alpha}} d t-\int_{u}^{s} \frac{(T-t)^{\alpha}-(s-t)^{\alpha}}{|t-u|^{\alpha}} d t \\
= & -\left.\frac{(u-t)^{1-\alpha}\left((T-t)^{\alpha}-(s-t)^{\alpha}\right)}{1-\alpha}\right|_{0} ^{u} \\
& -\left.\frac{(t-u)^{1-\alpha}\left((T-t)^{\alpha}-(s-t)^{\alpha}\right)}{1-\alpha}\right|_{u} ^{s} \\
& -\frac{\alpha}{1-\alpha} \int_{0}^{u}(u-t)^{1-\alpha}\left((T-t)^{\alpha}-(s-t)^{\alpha}\right) d t \\
& -\frac{\alpha}{1-\alpha} \int_{0}^{u}(t-u)^{1-\alpha}\left((T-t)^{\alpha}-(s-t)^{\alpha}\right) d t \\
= & \frac{(u-t)^{1-\alpha}\left(T-s^{\alpha}\right)}{1-\alpha}-\frac{(s-u)^{1-\alpha}(T-s)^{\alpha}}{1-\alpha} \\
& -\frac{\alpha}{1-\alpha} \int_{0}^{u}(u-t)^{1-\alpha}\left((T-t)^{\alpha-1}-(s-t)^{\alpha-1}\right) d t \\
& -\frac{\alpha}{1-\alpha} \int_{0}^{u}(t-u)^{1-\alpha}\left((T-t)^{\alpha-1}-(s-t)^{\alpha-1}\right) d t .
\end{aligned}
$$

Последнее выражение уже можно дифференцировать по $u$. В результате получим

$$
\begin{aligned}
K_{1}(T, u)-K_{1}(s, u)= & \frac{T^{\alpha}-s^{\alpha}}{u^{\alpha}}+\frac{(T-s)^{\alpha}}{(s-u)^{\alpha}} \\
& -\alpha \int_{0}^{u} \frac{(T-t)^{\alpha-1}-(s-t)^{\alpha-1}}{(u-t)^{\alpha}} d t \\
& +\alpha \int_{u}^{s} \frac{(T-t)^{\alpha-1}-(s-t)^{\alpha-1}}{(t-u)^{\alpha}} d t
\end{aligned}
$$


Замена $\frac{u-t}{u}=t_{1}$ дает

$$
\begin{aligned}
\int_{0}^{u} \frac{\left((T-t)^{\alpha-1}-(s-t)^{\alpha-1}\right)}{(u-t)^{\alpha} d t} & =\int_{0}^{u} \frac{\left(\frac{T-u}{u}+t_{1}\right)^{\alpha-1}-\left(\frac{s-u}{u}+t_{1}\right)^{\alpha-1}}{t_{1}^{\alpha}} d t_{1} \\
& =\int_{0}^{\frac{u}{T-u}} \frac{(1+t)^{\alpha-1}}{t^{\alpha}} d t-\int_{0}^{\frac{u}{s-u}} \frac{(1+t)^{\alpha-1}}{t^{\alpha}} d t \\
& =-\int_{\frac{u}{T-u}}^{\frac{u}{s-u}} \frac{(1+t)^{\alpha-1}}{t^{\alpha}} d t
\end{aligned}
$$

и замена $\frac{t-u}{s-u}=t_{1}$ дает

$$
\begin{aligned}
\int_{u}^{s} \frac{(T-t)^{\alpha-1}-(s-t)^{\alpha-1}}{(t-u)^{\alpha}} d t & =\int_{0}^{1} \frac{\left(\frac{T-u}{s-u}-t_{1}\right)^{\alpha-1}-\left(1-t_{1}\right)^{\alpha-1}}{t_{1}^{\alpha}} d t_{1} \\
& =\int_{0}^{\frac{s-u}{T-u}} \frac{\left(1-t_{1}\right)^{\alpha-1}}{t_{1}^{\alpha}} d t_{1}-\int_{0}^{1} \frac{\left(1-t_{1}\right)^{\alpha-1}}{t_{1}^{\alpha}} d t_{1} \\
& =-\int_{\frac{s-u}{T-u}}^{1} \frac{\left(1-t_{1}\right)^{\alpha-1}}{t_{1}^{\alpha}} d t_{1} .
\end{aligned}
$$

Окончательно,

$$
\begin{aligned}
K_{1}(T, u)-K_{1}(s, u)= & \frac{T^{\alpha}-s^{\alpha}}{u^{\alpha}}+\frac{(T-s)^{\alpha}}{(s-u)^{\alpha}} \\
& +\alpha \int_{\frac{u}{T-u}}^{\frac{u}{s-u}} \frac{(1+t)^{\alpha-1}}{t^{\alpha}} d t-\alpha \int_{\frac{s-u}{T-u}}^{1} \frac{(1-t)^{\alpha-1}}{t^{\alpha}} d t .
\end{aligned}
$$

Мы можем теперь написать

$$
m_{1}\left(C_{\rho}\right)=\int_{0}^{s}\left(K(T, u)-K_{1}(s, u)\right) d \xi(u) .
$$

Как уже отмечалось, при $\alpha>0$ для написанного стохастического интеграла справедливы обычные формулы интегрирования по частям. Поэтому

$$
\begin{aligned}
m_{1}\left(C_{\rho}\right)= & \left.\frac{T^{\alpha}-s^{\alpha}}{u^{\alpha}} \xi(u)\right|_{0} ^{s}+\left.\frac{(T-s)^{\alpha}}{(s-u)^{\alpha}}(\xi(u)-\xi(s))\right|_{0} ^{s} \\
& +\left.\alpha(\xi(u)-\xi(s)) \int_{\frac{u}{T-u}}^{\frac{u}{s-u}} \frac{(1+t)^{\alpha-1}}{t^{\alpha}} d t\right|_{0} ^{s} \\
& -\left.\alpha(\xi(u)-\xi(s)) \int_{\frac{s-u}{T-u}}^{1} \frac{(1-t)^{\alpha-1}}{t^{\alpha}} d t\right|_{0} ^{s} \\
& +\alpha\left(T^{\alpha}-s^{\alpha}\right) \int_{0}^{s} \frac{\xi(u)}{u^{1+\alpha}} d u-\alpha(T-s)^{\alpha} \int_{0}^{s} \frac{\xi(u)-\xi(s)}{(s-u)^{\alpha+1}} d u \\
& +\alpha \int_{0}^{s} \frac{\xi(u)-\xi(s)}{u^{\alpha}}\left(\frac{s^{\alpha}}{s-u}-\frac{T}{T-u}\right) d u \\
& +\alpha \int_{0}^{s} \frac{(\xi(u)-\xi(s))(T-s)^{\alpha}}{(s-u)^{\alpha+1}(T-u)} d u
\end{aligned}
$$




$$
\begin{aligned}
= & \frac{T^{\alpha}-s^{\alpha}}{s^{\alpha}} a+\frac{(T-s)^{\alpha}}{s^{\alpha}} a-\alpha a \int_{\frac{s}{T}}^{1} \frac{(1-t)^{\alpha-1}}{t^{\alpha}} d t \\
& +\alpha\left(T^{\alpha}-s^{\alpha}\right) \int_{0}^{s} \frac{\xi(u)}{u^{1+\alpha}} d u-\alpha(T-s)^{\alpha} \int_{0}^{s} \frac{\xi(u)-\xi(s)}{(s-u)^{\alpha+1}} d u \\
& +\alpha \int_{0}^{s} \frac{\xi(u)-\xi(s)}{u^{\alpha}} d u\left(\frac{s^{\alpha}}{s-u}-\frac{T^{\alpha}}{T-u}\right) d u \\
& +\alpha(T-s)^{\alpha} \int_{0}^{s} \frac{\xi(u)-\xi(s)}{(s-u)^{\alpha}(T-u)} d u .
\end{aligned}
$$

При вьводе последней формулы мы воспользовались тем, что

$$
\lim _{u \rightarrow 0} \frac{\xi(u)}{u^{\alpha}}=\lim _{u \rightarrow s} \frac{\xi(u)-\xi(s)}{(s-u)^{\alpha}}=0 .
$$

Это следует из того, что $\xi(u)$ локально, в окрестности любой точки, удовлетворяют при любом $\gamma>0$ условию Гёльдера с показателем $\frac{1+\alpha}{2}-\gamma$. Можем теперь написать

$$
\begin{aligned}
m_{1}\left(C_{\rho}\right)= & \frac{T^{\alpha}-s^{\alpha}}{s^{\alpha}} a+\frac{(T-s)^{\alpha}}{s^{\alpha}} a-\alpha a \int_{\frac{s}{T}}^{1} \frac{(1-t)^{\alpha-1}}{t^{\alpha}} d t \\
& +\alpha\left(T^{\alpha}-s^{\alpha}\right) s^{\frac{1-\alpha}{2}} \frac{1}{s^{\frac{1-\alpha}{2}}} \int_{0}^{s} \frac{\xi(u)}{u^{1+\alpha}} d u \\
& +\alpha(T-s)^{\alpha} s^{\frac{1-\alpha}{2}} \frac{1}{s^{\frac{1-\alpha}{2}}} \int_{0}^{s} \frac{\xi(s)-\xi(u)}{(s-u)^{\alpha+1}} d u \\
& -\alpha T^{\alpha} s^{\frac{1-\alpha}{2}} \frac{1}{s^{\frac{1-\alpha}{2}}} \int_{0}^{s} \frac{\xi(s)-\xi(u)}{u^{\alpha}}\left(\frac{s^{\alpha}}{T^{\alpha}} \frac{1}{s-u}-\frac{1}{T-u}\right) d u \\
& -\alpha(T-s)^{\alpha} s^{\frac{1-\alpha}{2}} \frac{1}{s^{\frac{1-\alpha}{2}}} \int_{0}^{s} \frac{\xi(s)-\xi(u)}{(s-u)^{\alpha+1}(T-u)} d u .
\end{aligned}
$$

Далее,

$$
a \frac{(T-s)^{\alpha}}{s^{\alpha}}-\alpha a \int_{\frac{s}{T}}^{s} \frac{(1-T)^{\alpha-1}}{t^{\alpha}} d t=-\alpha a \int_{\frac{s}{T}}^{s} \frac{(1-T)^{\alpha-1}}{t^{\alpha}}\left(\frac{1}{t^{\alpha}}-1\right) d t .
$$

При $x=\frac{T}{s} \rightarrow 1$ последнее выражение ведет себя как $(x-1)^{\alpha+1}$, а при $x \rightarrow \infty$ оно стремится к положительному пределу. Точно так же

$$
\begin{aligned}
\frac{T^{\alpha}-s^{\alpha}}{s^{\alpha}} a+\alpha\left(T^{\alpha}\right. & \left.-s^{\alpha}\right) \int_{0}^{s} \frac{\xi(u)}{u^{1+\alpha}} d u \\
& =\alpha\left(T^{\alpha}-s^{\alpha}\right) \int_{0}^{1} \frac{\xi(u)}{u^{1+\alpha}} d u+\alpha\left(T^{\alpha}-s^{\alpha}\right) \int_{1}^{s} \frac{\xi(u)-\xi(s)}{u^{1+\alpha}} d u .
\end{aligned}
$$

Аналогичньм образом,

$$
\begin{aligned}
\alpha(T-s)^{\alpha} \int_{0}^{s} \frac{\xi(s)-\xi(u)}{(s-u)^{\alpha+1}} d u-\alpha(T-s)^{\alpha} \int_{0}^{s} \frac{\xi(s)-\xi(u)}{(s-u)^{\alpha}(T-u)} d u & \\
& =\alpha(T-s)^{\alpha+1} \int_{0}^{s} \frac{\xi(s)-\xi(u)}{(s-u)^{\alpha+1}(T-u)} d u
\end{aligned}
$$


и

$$
\begin{aligned}
\int_{0}^{s} \frac{\xi(s)-\xi(u)}{u^{\alpha}} & \left(\frac{s^{\alpha}}{T^{\alpha}} \frac{1}{s-u}-\frac{1}{T-u}\right) d u \\
& =\frac{T^{\alpha}-s^{\alpha}}{T^{\alpha}} \int_{0}^{s} \frac{\xi(s)-\xi(u)}{u^{\alpha}(s-u)} d u+(T-s) \int_{0}^{s} \frac{\xi(s)-\xi(u)}{u^{\alpha}(s-u)(T-u)} d u .
\end{aligned}
$$

Таким образом,

$$
\begin{aligned}
m_{1}\left(C_{\rho}\right)= & \alpha\left(T^{\alpha}-s^{\alpha}\right) \int_{0}^{1} \frac{\xi(u)}{u^{\alpha+1}} d u+\alpha\left(T^{\alpha}-s^{\alpha}\right) \int_{1}^{s} \frac{\xi(s)-\xi(u)}{u^{\alpha+1}} d u \\
& -\alpha a \int_{\frac{s}{T}}^{1}(1-t)^{\alpha-1}\left(\frac{1}{t^{\alpha}}-1\right) d t+\alpha(T-s)^{\alpha+1} \int_{0}^{s} \frac{\xi(s)-\xi(u)}{(s-u)^{\alpha+1}(T-u)} d u \\
& +\alpha\left(T^{\alpha}-s^{\alpha}\right) \int_{0}^{s} \frac{\xi(s)-\xi(u)}{u^{\alpha}(s-u)} d u-\alpha(T-s) T^{\alpha} \int_{0}^{s} \frac{\xi(s)-\xi(u)}{u^{\alpha}(s-u)(T-u)} d u .
\end{aligned}
$$

Исследуем вначале область малых $s$, т.е. $s \leqslant T / 2$. В этой области все слагаемые имеют одинаковый порядок. Мы воспользуемся нашими предположениями Н1), Н2) (см. $\S 1)$, которые дают

$$
\begin{aligned}
m_{1}\left(C_{\rho}\right)= & \alpha\left(T^{\alpha}-s^{\alpha}\right) \int_{0}^{1} \frac{\xi(u)}{u^{\alpha+1}} d u \\
& +\alpha\left(T^{\alpha}-s^{\alpha}\right) s^{\frac{1-\alpha}{2}} \frac{1}{s^{\frac{1-\alpha}{2}}} \int_{1}^{s} \frac{\xi(u)-\xi(s)}{u^{\alpha+1}} d u-\alpha a \int_{\frac{s}{T}}^{s}(1-t)^{\alpha-1}\left(\frac{1}{t^{\alpha}}-1\right) d t \\
& +\alpha(T-s)^{\alpha+1} s^{\frac{1-\alpha}{2}} \frac{1}{s^{\frac{1-\alpha}{2}}} \int_{0}^{s} \frac{\xi(u)-\xi(s)}{(s-u)^{\alpha+1}}(T-u) d u \\
& +\alpha\left(T^{\alpha}-s^{\alpha}\right) s^{\frac{1-\alpha}{2}} \frac{1}{s^{\frac{1-\alpha}{2}}} \int_{0}^{s} \frac{\xi(u)-\xi(s)}{u^{\alpha}(s-u)} d u \\
& -\alpha T^{\alpha} s^{\frac{1-\alpha}{2}} \frac{1}{s^{\frac{1-\alpha}{2}}} \int_{0}^{s} \frac{\xi(u)-\xi(s)}{u^{\alpha}(s-u)} \frac{T-s}{T-u} d u \\
= & T^{\alpha} s^{\frac{1-\alpha}{2}} \eta_{1},
\end{aligned}
$$

где $\eta_{1}$ - случайная величина, $\mathrm{E} \eta_{1}=O(1)$. Поэтому

$$
\frac{m_{1}\left(C_{\rho}\right)}{\sqrt{\sigma(s, T)}}=\frac{1}{T^{\frac{1-\alpha}{2}}} s^{\frac{1-\alpha}{2}} \eta_{2} .
$$

Аналогичньм образом,

$$
\frac{\partial m_{1}\left(C_{\rho}\right)}{\partial T}=T^{\alpha-1} s^{\frac{1-\alpha}{2}} \eta_{3}, \quad \mathrm{E}_{s} \eta_{3}=O(1)
$$

и

$$
\frac{\sigma_{T}}{\sigma} \frac{m_{1}}{\sqrt{\sigma}}-\frac{\partial m_{1}}{\partial T} \frac{1}{\sqrt{\sigma}}=\frac{1}{T^{\frac{3-\alpha}{2}}} s^{\frac{1-\alpha}{2}} \eta_{3}, \quad \mathrm{E} \eta_{3}=O(1) .
$$

Аналогично исследуется $m_{2}\left(C_{\rho}\right)$. 
Поскольку (см. (7))

$$
\Phi_{T}(s, T)=\int e^{-\frac{m^{2}\left(C_{\rho}\right)}{2 \sigma(s, T)}} \frac{1}{\sqrt{2 \pi}}\left(\frac{1}{\sigma(s, T)} \cdot \frac{\partial \sigma(s, T)}{\partial T} \cdot \frac{m\left(C_{\rho}\right)}{\sqrt{\sigma(s, T)}}-\frac{1}{\sqrt{\sigma(s, T)}} \cdot \frac{\partial m\left(C_{\rho}\right)}{\partial T}\right)
$$

то мы получаем, что при $s \leqslant T / 2$

$$
\left|\Phi_{T}(s, T)\right| \leqslant \text { const } \cdot \alpha \cdot \frac{s^{\frac{1-\alpha}{2}}}{T^{\frac{3-\alpha}{2}}} .
$$

Исследование $m_{1}\left(C_{\rho}\right)$ при $s>T / 2$ более сложно. Здесь мы воспользуемся гипотезой $\mathrm{H} 3)$ и некоторыми сокрашениями. При рассматриваемых $s$ условное математическое ожидание $m_{1}\left(C_{\rho}\right)$ принимает значения порядка $\sqrt{\sigma(s, T)}$, и мы выделим главньй член в представлении $m_{1}\left(C_{\rho}\right)$. Имеем

$$
\begin{aligned}
m_{1}(s)= & \int m_{1}\left(C_{\rho}\right) d P\left(C_{\rho} ; s\right) \\
= & \alpha\left(T^{\alpha}-s^{\alpha}\right) \int_{0}^{1} \frac{g_{1}(u, s)}{u^{\frac{1+\alpha}{2}}} d u+\alpha\left(T^{\alpha}-s^{\alpha}\right) \int_{0}^{1} \frac{g_{1}(u, s)-a u^{-\frac{1+\alpha}{2}}}{u^{\frac{1+\alpha}{2}}} d u \\
& -\alpha a \int_{s / T}^{1}(1-t)^{\alpha-1}\left(\frac{1}{t^{\alpha}}-1\right) d t+\alpha(T-s)^{\alpha+1} \int_{0}^{s} \frac{g_{2}(u, s) d u}{(s-u)^{\frac{\alpha+1}{2}}(T-u)} \\
& +\alpha\left(T^{\alpha}-s^{\alpha}\right)(T-s) \int_{0}^{s} \frac{g_{2}(u, s) d u}{u^{\alpha}(s-u)^{\frac{1-\alpha}{2}}}-\alpha T^{\alpha} \int_{0}^{s} \frac{g_{2}(u, s) d u}{u^{\alpha}(s-u)^{\frac{1-\alpha}{2}}} .
\end{aligned}
$$

Максимальным (при $(T-s) / s \rightarrow 0)$ слагаемым в этом представлении служит

$$
J=\alpha(T-s)^{\alpha+1} \int_{0}^{s} \frac{g_{2}(u, s) d u}{(s-u)^{\frac{1-\alpha}{2}}(T-u)} .
$$

Перепишем его следуюшим образом:

$$
\begin{aligned}
J= & \alpha(T-s)^{\alpha+1} g_{2}(s, s) \int_{0}^{s} \frac{d u}{(s-u)^{\frac{\alpha+1}{2}}(T-u)} \\
& +\alpha(T-s)^{\alpha+1} \int_{0}^{s} \frac{\left(g_{2}(u, s)-g_{2}(s, s)\right) d u}{(s-u)^{\frac{\alpha+1}{2}}(T-u)} \\
= & \alpha(T-s)^{\frac{\alpha+1}{2}} \int_{0}^{\frac{s}{T-s}} \frac{d u}{(s-u)^{\frac{\alpha+1}{2}}(1+u)} \\
& +\alpha(T-s)^{\alpha-1} \int_{0}^{s} \frac{\left(g_{2}(u, s)-g_{2}(s, s)\right) d u}{(s-u)^{\frac{\alpha+1}{2}}(T-u)} .
\end{aligned}
$$


Таким образом,

$$
\begin{aligned}
m_{1}(s)= & \alpha(T-s)^{\frac{\alpha+1}{2}} g_{2}(s, s) \int_{0}^{\frac{1}{x-1}} \frac{d u}{u^{\frac{\alpha+1}{2}}(u+1)} \\
& +\alpha(T-s)^{\alpha+1} \int_{0}^{s} \frac{\left(g_{2}(u, s)-g_{2}(s, s)\right) d u}{(s-u)^{\frac{\alpha+1}{2}}(T-u)} \\
& +\alpha\left(T^{\alpha}-s^{\alpha}\right)\left[\int_{0}^{1} \frac{g_{1}(u, s)}{u^{\frac{\alpha+1}{2}}} d u+\int_{1}^{s} \frac{g_{1}(u, s)-a u^{-\frac{1+\alpha}{2}}}{u^{\frac{1+\alpha}{2}}} d u\right] \\
& -\alpha a \int_{s / T}^{1}(1-t)^{1-\alpha}\left(\frac{1}{t^{\alpha}}-1\right) d t \\
& +\alpha\left(T^{\alpha}-s^{\alpha}\right)(T-s) \int_{0}^{s} \frac{g_{2}(u, s)}{u^{\alpha}(s-u)} d u-\alpha T^{\alpha}(T-s) \int_{0}^{s} \frac{g_{2}(u, s)}{u^{\alpha}(s-u)^{\frac{1-\alpha}{2}}} d u .
\end{aligned}
$$

Теперь видим, что отношение $\frac{m_{1}(s)}{\sqrt{\sigma(s, T)}}$ имеет вид

$$
\begin{aligned}
\frac{m_{1}(s)}{\sqrt{\sigma(s, T)}=} & \frac{\alpha g_{2}(s, s)}{\sqrt{\psi(T / s)}}+\frac{\alpha(T-s)^{\frac{\alpha+1}{2}}}{\psi_{1}(T / s)} \int_{0}^{s} \frac{\left(g_{2}(u, s)-g_{2}(s, s)\right) d u}{(s-u)^{\frac{\alpha+1}{2}}(T-u)} \\
& +\frac{\alpha\left(T^{\alpha}-s^{\alpha}\right)}{(T-s)^{\frac{\alpha+1}{2}} \sqrt{\psi_{1}(T / s)}}\left[\int_{0}^{1} \frac{g_{1}(u, s) d u}{u^{\frac{1+\alpha}{2}}}+\int_{1}^{s} \frac{g_{1}(u, s)-a u^{-\frac{1+\alpha}{2}}}{u^{\frac{1+\alpha}{2}}} d u\right] \\
& -\frac{\alpha a}{(T-s)^{\frac{1+\alpha}{2}} \sqrt{\psi(T / s)}} \int_{s / T}^{1}(1-t)^{1-\alpha}\left(\frac{1}{t^{\alpha}}-1\right) d t \\
& +\frac{\alpha\left(T^{\alpha}-s^{\alpha}\right)(T-s)^{\frac{1-\alpha}{2}}}{\sqrt{\psi_{1}(T / s)}} \int_{0}^{s} \frac{g_{2}(u, s) d u}{u^{\alpha}(s-u)} \\
& -\frac{\alpha T^{\alpha}(T-s)^{\frac{1-\alpha}{2}}}{\sqrt{\psi_{1}(T / s)}} \int_{0}^{s} \frac{g_{2}(u, s) d u}{u^{\alpha}(s-u)^{\frac{1-\alpha}{2}}}
\end{aligned}
$$

В этой формуле существенно, что первое слагаемое зависит от $T$ только через регулярную функцию $\psi_{1}(T / s)$, а остальные слагаемые при малых $(T-s)$ пропорциональны $(T-s)^{\frac{1-\alpha}{2}},(T-s)^{\frac{3-\alpha}{2}}, \ldots$ Поэтому основной вклад в производную $\frac{\partial}{\partial T} \frac{m_{1}(s)}{\sqrt{\sigma(s, T)}}$
вносит производная второго слагаемого, равная

$$
\begin{aligned}
& \alpha \cdot \frac{\alpha+1}{2} \frac{(T-s)^{-\frac{1-\alpha}{2}}}{\sqrt{\psi_{1}(T / s)}} \int_{0}^{s} \frac{\left(g_{2}(u, s)-g_{2}(s, s)\right) d u}{(s-u)^{\frac{\alpha+1}{2}}(T-u)} \\
&+\alpha \frac{(T-s)^{\frac{\alpha+1}{2}}}{\sqrt{\psi_{1}(T / s)}} \int_{0}^{s} \frac{\left(g_{2}(u, s)-g_{2}(s, s)\right) d u}{(s-u)^{\frac{\alpha+1}{2}}(T-u)^{2}} \\
& \quad-\frac{\alpha}{2} \cdot \frac{(T-s)^{-\frac{1-\alpha}{2}}}{\left(\psi_{1}(T / s)\right)^{\frac{3}{2}}} \cdot \frac{\psi_{1}^{\prime}(T / s)}{s} \int_{0}^{s} \frac{\left(g_{2}(u, s)-g_{2}(s, s)\right)}{(s-u)^{\frac{\alpha+1}{2}}(T-u)^{2}} d u
\end{aligned}
$$


Здесь для оценки всех интегралов мы должны воспользоваться гипотезами Н2), Н3), из которых следует, что при $T-s \leqslant 1$

$$
\begin{aligned}
& \left|\int_{0}^{s} \frac{g_{2}(u, s)-g_{2}(s, s)}{(s-u)^{\frac{\alpha+1}{2}}(T-u)^{2}} d u\right| \leqslant \frac{\text { const }}{(T-s)^{\frac{\alpha+1}{2}-\gamma} s^{\gamma}} \\
& \left|\int_{0}^{s} \frac{g_{2}(u, s)-g_{2}(s, s)}{(s-u)^{\frac{\alpha+1}{2}}(T-u)^{2}} d u\right| \leqslant \frac{\mathrm{const}}{(T-s)^{\frac{3+\alpha}{2}-\gamma} s^{\gamma}}
\end{aligned}
$$

а при $T-s>1$ с помошю неравенства $\left|g_{2}(u, s)-g_{2}(s, s)\right| \leqslant$ const $\left|\frac{s-u}{s}\right|$ получаем

$$
\begin{aligned}
& \left|\int_{0}^{s} \frac{g_{2}(u, s)-g_{2}(s, s)}{(s-u)^{\frac{\alpha+1}{2}}(T-u)} d u\right| \leqslant \frac{\text { const }}{(T-s)^{\frac{\alpha+1}{2}-\gamma} s^{\gamma}} \\
& \left|\int_{0}^{s} \frac{g_{2}(u, s)-g_{2}(s, s)}{(s-u)^{\frac{\alpha+1}{2}}(T-u)^{2}} d u\right| \leqslant \frac{\mathrm{const}}{(T-s)^{\frac{\alpha+3}{2}-\gamma} s^{\gamma}} .
\end{aligned}
$$

Таким образом, при $T-s \leqslant s$

$$
\mathrm{E}_{s} e^{-\frac{m^{2}\left(C_{\rho}\right)}{2 \sigma(s, T)}}\left|\frac{\partial}{\partial T} \frac{m_{1}\left(C_{\rho}\right)}{\sqrt{\sigma(s, T)}}\right| \leqslant \frac{\text { const } \cdot \alpha}{(T-s)^{1-\gamma} s^{\gamma}} .
$$

Интеграл от правой части по $T$ от $T=s$ до $T=2 s$ не превосходит const $\cdot \alpha$.

Аналогично исследуется $m_{2}\left(C_{\rho}\right)$.

\section{§. Окончание доказательства основной теоремы.}

Заключительные замечания

Рассмотрим вначале $\Phi(s, s)=\int d P\left(C_{\rho}, T\right) \lim _{T \rightarrow s} \int_{0}^{-\frac{m\left(C_{\rho}\right)}{\sqrt{\sigma(s, T)}}} \frac{1}{\sqrt{2 \pi}} e^{-\frac{u^{2}}{2}} d u$.

В силу гипотез Н2), Н3)

$$
\begin{aligned}
\lim _{T \rightarrow s} \frac{m_{1}\left(C_{\rho}\right)}{\sqrt{\sigma(s, T)}} & =\alpha \cdot \lim _{T \rightarrow s}(T-s)^{\frac{\alpha+1}{2}} \int_{0}^{s} \frac{\mathrm{E}_{s}(\xi(s)-\xi(u))}{(s-u)^{\alpha+1}(T-u)^{2}} d u \\
& =\alpha \cdot \lim _{T \rightarrow s}(T-s)^{\frac{\alpha+1}{2}} \int_{0}^{s} \frac{g(u, s)}{(s-u)^{\alpha+1}(T-u)^{2}} d u \\
& =\alpha \cdot \lim _{T \rightarrow s}(T-s) \int_{0}^{\frac{s}{T-s}} \frac{g(s-v(T-s), s)}{v^{\frac{\alpha+1}{2}}(1+v)} d v \\
& =\alpha g_{2}(s, s) \int_{0}^{\infty} \frac{d v}{v^{\frac{\alpha+1}{2}}(1+v)}
\end{aligned}
$$

Ввиду наших предположений $g_{2}(s, s)$ равномерно ограничена. Аналогично исследуется $\lim _{T \rightarrow s} \frac{m_{2}\left(C_{\rho}\right)}{\sqrt{\sigma(s, T)}}$.

Таким образом, $\Phi(T, T)$ равномерно меньше 1. С вероятностной точки зрения

$$
\lim _{T \rightarrow s} \frac{m\left(C_{\rho}\right)}{\sqrt{\sigma(s, T)}}
$$


представляет собой “инфинитезимальный прогноз”, которьй оказьвается нетривиальньм.

Полученные оценки $\Phi_{T}(s, T)$ показывают, что

$$
\int_{0}^{T}\left|\Phi_{T}(s, T)\right| d s \leqslant \text { const } \cdot \alpha .
$$

Поэтому при малых $\alpha$ основное уравнение (5) можно решать методом итераций. Прямая оценка возникающего ряда дает оценку (8).

Для получения окончательного результата необходимо доказать гипотезы Н1)-H3). Из них гипотеза Н3) представляется наиболее трудной.

\section{СПИСОК ЛИТЕРАТУРЫ}

[1] Колмогоров А. Н. Спираль Винера и некоторые другие интересные кривые в гильбертовом пространстве // Докл. АН СССР. 1940. Т. 26. №2. С. 115-118.

[2] Леви П. Стохастические процессы и броуновское движение. М.: Наука, 1972.

[3] Mandelbrot B. B. The fractional geometry of nature. San Francisco: Freeman, 1982.

[4] Kahane J.-P. Some random series of functions. Cambridge Stud. Adv. Math. V. 5. Cambridge: Cambridge Univ. Press, 1985.

[5] Talagrand M. Lower classes for fractional Brownian motion // J. Theoret. Probab. 1996. V. 9. № 1. P. 191-213.

[6] Yor M. Remarques sur certaines constructions des mouvements browniens fractionnaires // Lecture Notes in Math. V. 1321. Berlin: Springer-Verlag, 1988. P. 217-224.

[7] Синай Я. Г. Теория фазовых переходов. М.: Наука, 1980.

[8] Dobrushin R. L. Gaussian and their subordinated self-similar random generalized fields // Ann. Probab. 1979. V. 7. №1. P. 1-28.

[9] Dobrushin R. L., Major P. On asymptotical behavior of some self-similar random fields // Selecta Math. Soviet. 1981. V. 1. № 5. P. 256-291.

[10] Patzschke N., Zaehle M. Random measures IV // Stochastics and Stoch. Reports. 1994. V. 49. P. 87-98.

[11] Самко С.Г., Килбас А.А., Маричев О.И. Интегралы и производные дробного порядка и некоторые их приложения. Минск: Наука и техника, 1987.

Отделение математики Принстонского Университета,

Институт теоретической физики им. Л. Д. Ландау РАН

Поступила в редакцию

15.09.1996 